\title{
EDITORIAL
}

\section{Comment on "At-risk drinking and current cannabis use among medical students: a multivariable analysis of the role of personality traits"}

\author{
Scott D. Lane iD \\ Louis A. Faillace, MD, Department of Psychiatry and Behavioral Sciences, McGovern School of Medicine, The University of Texas Health \\ Science Center at Houston (UTHealth), Houston, TX, USA. MD Anderson UTHealth Graduate School of Biomedical Sciences, Houston, \\ TX, USA.
}

The paper by Schwarzbold et al. ${ }^{1}$ comments on the relatively unique population of medical students, which differs from the broader general population on key factors such as general intelligence, academic and career motivation, and self-selection to a helping profession. Thus, the data need to be understood in proper context. In general, the observed associations between the Five Factor Model of Personality (Big Five), Behavioral Inhibition and Activation Systems scales (BIS/BAS) and alcohol and marijuana use are generally consistent with the existing literature in the broader population, and consistent with findings from other countries and continents. However, some findings from this report are equivocal or do not support the broader literature regarding some specific subscales of the Big Five and BIS/BAS. Additionally, the results are partially complicated by a lack of observed association between depression and anxiety scores and the Patient Health Questionnaire (PHQ-4), which might be expected in a sufficient sample size (here, $n>700$ ). However, the authors appropriately raise the possibility that the lack of measurement sensitivity in the screening instruments may have played a role in the observed results. Overall, then, generalizing outside this medical school population to the broader population may be premature. It might, however, suggest there are unique profiles within medical-student populations (vs. the general population), or perhaps more conservatively, within medical students in southern Brazil.

The selection of the primary instruments in this study was carefully considered, as both the Big Five and BIS/ BAS are well validated. Personality factors - including neuroticism and high BAS score - can indicate risk for the development of alcohol and cannabis use disorders, ${ }^{2}$ as well as other illicit drugs of abuse, i.e. cocaine. ${ }^{3}$

The authors were careful not to use causal language and to discuss their findings in terms of associations with the variables of interest, noting that the study was exploratory. The results are properly interpreted as correlational and (where appropriate) exploratory. Notably, this does not negate the value of employing sociodemographic and psychometric measures as predictive tools to aid in the examination of risky behavior patterns, both in the general population and, as in the present case, specific populations. Such inquiries can help inform the construction of screening tools in health surveys and subsequent interventions. ${ }^{4}$

With regard to the measurement approach, a single omnibus multivariate model for the data analyses might have provided more parsimony both in computation and interpretation. Given the sample size, there appears to be adequate power to construct a single model. However, it appears unlikely it would change the primary results or conclusions to be gleaned from the report. With additional regard to measurement, the author's provide adjusted odds ratio (AOR), which can be understood as a proxy for effect size. It should be recognized that different domains of inquiry across the applied sciences have different standards for the interpretation of "meaningful" effects sizes. Providing some context for these odds ratios with regard to prior work in this area may aid interpretation and prove helpful for readers interested in this research domain. ${ }^{5,6}$

The authors correctly note that sociodemographic factors may be important in differentiating risky behavior patterns among groups of medical students. Future work may do well to collect more in-depth and diverse sociodemographic information. Another avenue for future investigation may include quantification of alcohol and marijuana use patterns. In the present report, data from the screening instruments were used to create binary variables (yes/no on Alcohol Use Disorders Identification Test-Consumption [AUDIT-C] and 30-day cannabis use). More granular quantification of drug use patterns measures of alcohol and cannabis as continuous rather than binary variables - may provide critical information to enable more informative estimates via linear or nonlinear modeling. Efforts to enhance the predictive utility of screening tools may also be furthered by adding variables 
known to be strong predictors of substance misuse, for example family history of substance abuse, history of trauma, and age of first substance use. These are relatively easy to assess in the context of survey research. Notably, the present report provides an excellent starting point from which to launch a systematic line of investigation, with potential for applied utility.

The authors properly consider the study limitations, and also make useful suggestions for future investigations in this area. As a first approximation, these data might prove useful in the development of screening tools for the identification of medical students who are in need of support services to motivate and guide healthy behavior patterns as they navigate the often challenging transition into and through medical school education. ${ }^{7}$

\section{Disclosure}

The author reports no conflicts of interest.

\section{References}

1 Schwarzbold ML, Haas GM, Barni RS, Biava P, Momo AC, Dias TM, et al. At-risk drinking and current cannabis use among medical students: a multivariable analysis of the role of personality traits. Braz J Psychiatry. 2020;42:136-44.

2 Dash GF, Slutske WS, Martin NG, Statham DJ, Agrawal A, Lynskey MT. Big Five personality traits and alcohol, nicotine, cannabis, and gambling disorder comorbidity. Psychol Addict Behav. 2019;33:420-9.

3 Balconi M, Finocchiaro R, Campanella S. Reward sensitivity, decisional bias, and metacognitive deficits in cocaine drug addiction. J Addict Med. 2014;8:399-406.

4 Clinton-McHarg T, Yoong SL, Tzelepis F, Regan T, Fielding A, Skelton E, et al. Psychometric properties of implementation measures for public health and community settings and mapping of constructs against the consolidated framework for implementation research: a systematic review. Implement Sci. 2016;11:148.

5 Schafer T, Schwartz MA. The meaningfulness of effect sizes in psychological research: Differences between sub-disciplines and the impact of potential biases. Front Psychol. 2019;10:813.

6 lalongo C. Understanding the effect size and its measures. Biochem Med (Zagreb). 2016;26:150-63.

7 Gentile JP, Roman B. Medical student mental health services: psychiatrists treating medical students. Psychiatry (Edgemont). 2009;6: 38-45. 